\title{
Editorial
}

\section{Gender Affirmation Surgery}

\author{
Gennaro Selvaggi $\left(\mathbb{D},{ }^{1}\right.$ Christopher J. Salgado ${ }^{(D)},{ }^{2}$ Stan Monstrey $\mathbb{D D}^{3}{ }^{3}$ \\ and Miroslav Djordevic $\mathbb{D}^{4,5}$ \\ ${ }^{1}$ Department of Plastic Surgery, Institute of Clinical Sciences, Sahlgrenska Academy, University of Gothenburg, \\ Sahlgrenska University Hospital, Gothenburg, Sweden \\ ${ }^{2}$ Division of Plastic Surgery, University of Miami Miller School of Medicine, Miami, FL, USA \\ ${ }^{3}$ Department of Plastic Surgery, University Hospital Gent, Gent, Belgium \\ ${ }^{4}$ Belgrade Center for Genital Reconstructive Surgery, Belgrade, Serbia \\ ${ }^{5}$ School of Medicine, University of Belgrade, Belgrade, Serbia \\ Correspondence should be addressed to Gennaro Selvaggi; selvaggigennaro@yahoo.it
}

Received 4 June 2018; Accepted 5 June 2018; Published 5 July 2018

Copyright (C) 2018 Gennaro Selvaggi et al. This is an open access article distributed under the Creative Commons Attribution License, which permits unrestricted use, distribution, and reproduction in any medium, provided the original work is properly cited.

Gender Affirmation Surgery (GAS) is a collection of surgical procedures performed in patients presenting with diagnosis of "Gender Dysphoria" (according to the Diagnostic Statistical Manual of Mental Disorders (DSM-V), published by the American Psychiatric Association [1] (2013)) or "Transsexualism" (according to the International Classification of Diseases (ICD-10), published by the World Health Organization $[2,3]$ (WHO, 1992; WHO, 2007)).

Gender Dysphoria (GD) and Transsexualism (T) are both referring to the "discomfort or distress caused by the discrepancy between a person's gender identity and that person's sex assigned at birth" [4, 5] (Fisk, 1974; Knudson, De Cuypere, and Bockting, 2010). Mental health professionals are in charge of making a diagnosis; however as surgeons operating on patients we need to agree with the diagnosis prior to our interventions.

The treatment for GD may consist of mental health therapy, cross-sex hormone therapy, and different forms of surgery. More specifically, GAS refers to the whole genital, facial, and body procedures required to create a body phenotype that best represents one's own identity. The requested body phenotype is not always representing a fully masculine or feminine aspect, and this may be representative of patient satisfaction of their desired goals in transition. Many individuals, in fact, might opt to undergo only some of the surgical procedures currently available, while others opt to receive the full collection of treatments. Those who are requesting only part of the collection of procedures, for example, transmen seeking mastectomy and not penile construction, might do so either because they represent a gender nonconforming identity or because they realize that the surgical technique(s) available will not fulfill their expectations.

Indeed, there are also individuals that, although having discrepancy between one's own gender and that assigned at birth, might experience only a minimum distress, or no distress at all, and therefore they might opt for other treatments (mental health therapy and/or hormonal therapy) but surgery, or no treatment at all.

To date, the majority of the academic centers worldwide are managing this condition according to the Standards of Care [6] as proposed by the World Professional Association of Transgender Health.

Gender Affirmation Surgery remains an area of superspecialization and is in constant development. Few surgical refinements have been published within the last few years, and follow-ups of long-term series of patients proceed slowly. Cooperation among centers represents a solution to standardize approaches and techniques and achieves a higher level of evidence. This cooperation is realized through reciprocal visiting and meetings, discussion during conferences, and live-surgery sessions in order to learn "the way he/she does it". At the same time, multicenter studies are to be promoted, in order to collect large series of cases that would add to the evidence of a given procedure. Finally, 
standardized and validated methods of assessment need to be defined (Patient Reported Outcome Measures) [7, 8], in order to measure objectively, without bias, the outcomes of each treatment.

Lastly, the development of medical and surgical techniques must advance in parallel to ethical discussions on the permissibility of specific treatments, research protocols, or more innovative surgeries. In fact, the large variability of the transgender population (considering both one's experience of the gender identity and the relationship within one's sociocultural background) poses ethical questions that often go beyond medical ethics and extend to areas of sociopolitical sciences and human rights. In this issue, we highlight scientific advances made in the field of Gender Affirmation Surgery with an international collaborative approach that allows this issue to be both comprehensive and informative.

Gennaro Selvaggi
Christopher J. Salgado
Stan Monstrey
Miroslav Djordevic

\section{References}

[1] J. Talairach and P. Thournoux, Diagnostic and Statistical Manual of Mental Disorders, Washington, 5th edition, 2013.

[2] World Health Organization, International classification of diseases and related health problems - 10th revision, WHO, Geneva, Switzerland, 2007.

[3] World Health Organization, Manual of the International Statistical Classification of Diseases, Injuries, and Causes of Death, WHO, Geneva, Switzerland, Tenth Revision edition, 1992.

[4] N. M. Fisk, "Gender Dysphoria Syndrome-the conceptualization that liberalizes indication for total gender reorientation and implies a broadly based multi-dimensional rehabilitative regimen," Western Journal of Medicine, vol. 120, no. 5, pp. 386391, 1974.

[5] G. Knudson, G. de Cuypere, and W. Bockting, "Recommendations for revision of the DSM diagnoses of gender identity disorders: Consensus statement of the world professional association for transgender health," International Journal of Transgenderism, vol. 12, no. 2, pp. 115-118, 2010.

[6] WPATH, Standards of Care for the Health of Transsexual, Transgender, and Gender Nonconforming People, WPATH, 7th edition, 2011.

[7] M. Andréasson, K. Georgas, A. Elander, and G. Selvaggi, "Patient-Reported Outcome Measures Used in Gender Confirmation Surgery," Plastic and Reconstructive Surgery, vol. 141, no. 4, pp. 1026-1039, 2018.

[8] M. Barone, A. Cogliandro, N. Di Stefano, V. Tambone, and P. Persichetti, "A Systematic Review of Patient-Reported Outcome Measures Following Transsexual Surgery," Aesthetic Plastic Surgery, vol. 41, no. 3, pp. 700-713, 2017. 


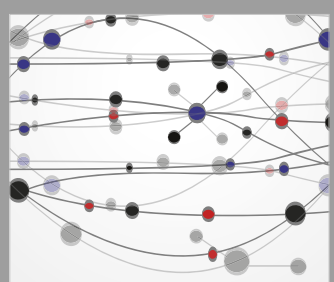

The Scientific World Journal
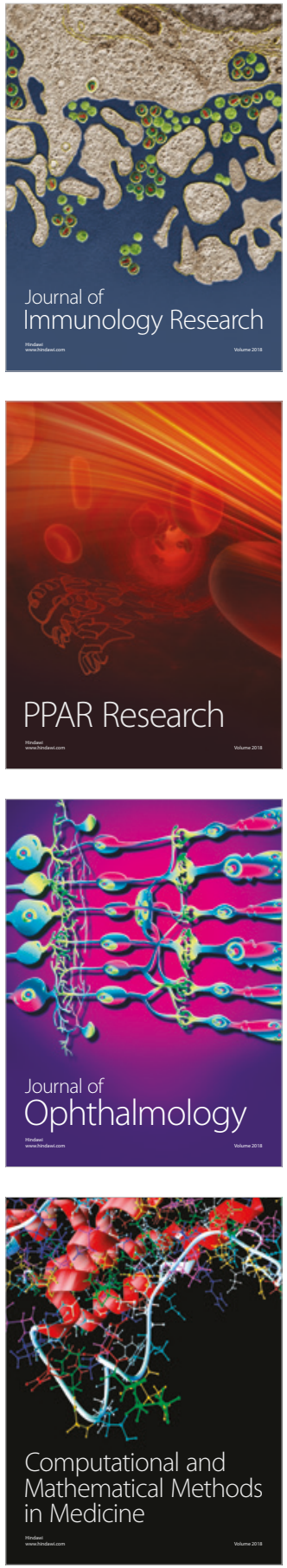

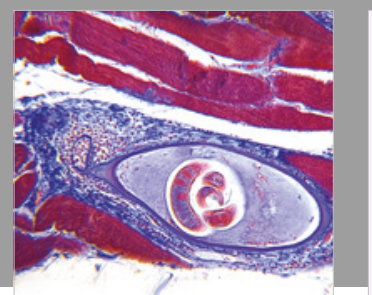

Gastroenterology Research and Practice

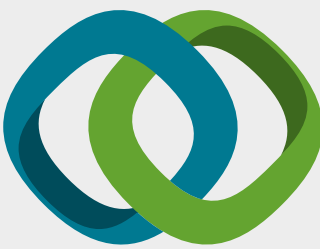

\section{Hindawi}

Submit your manuscripts at

www.hindawi.com
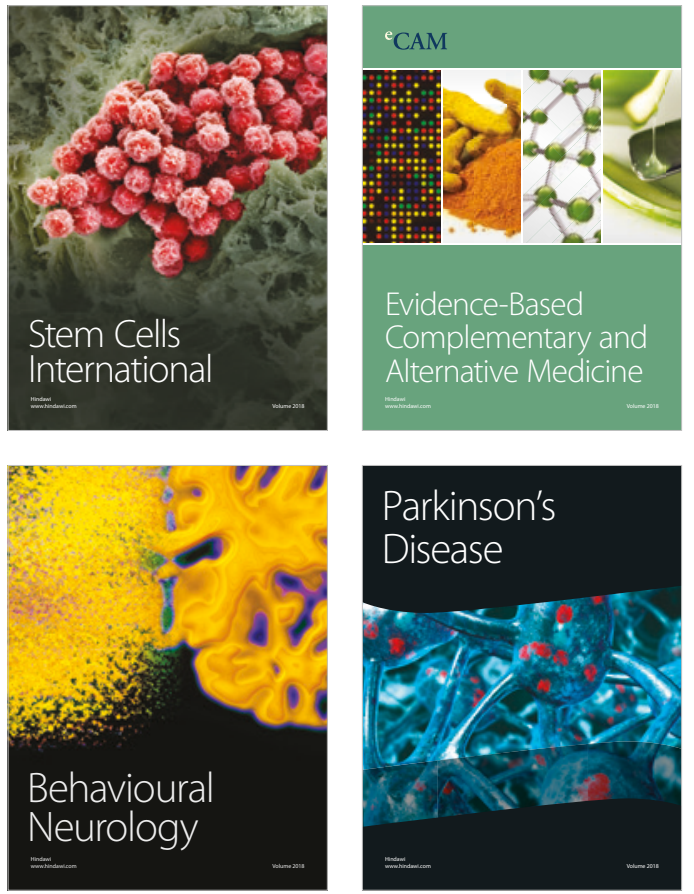

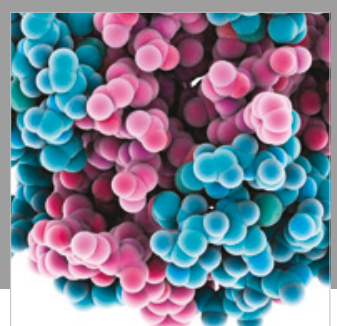

ournal of

Diabetes Research

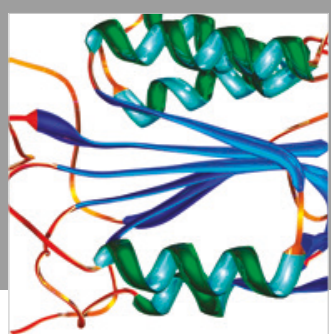

Disease Markers
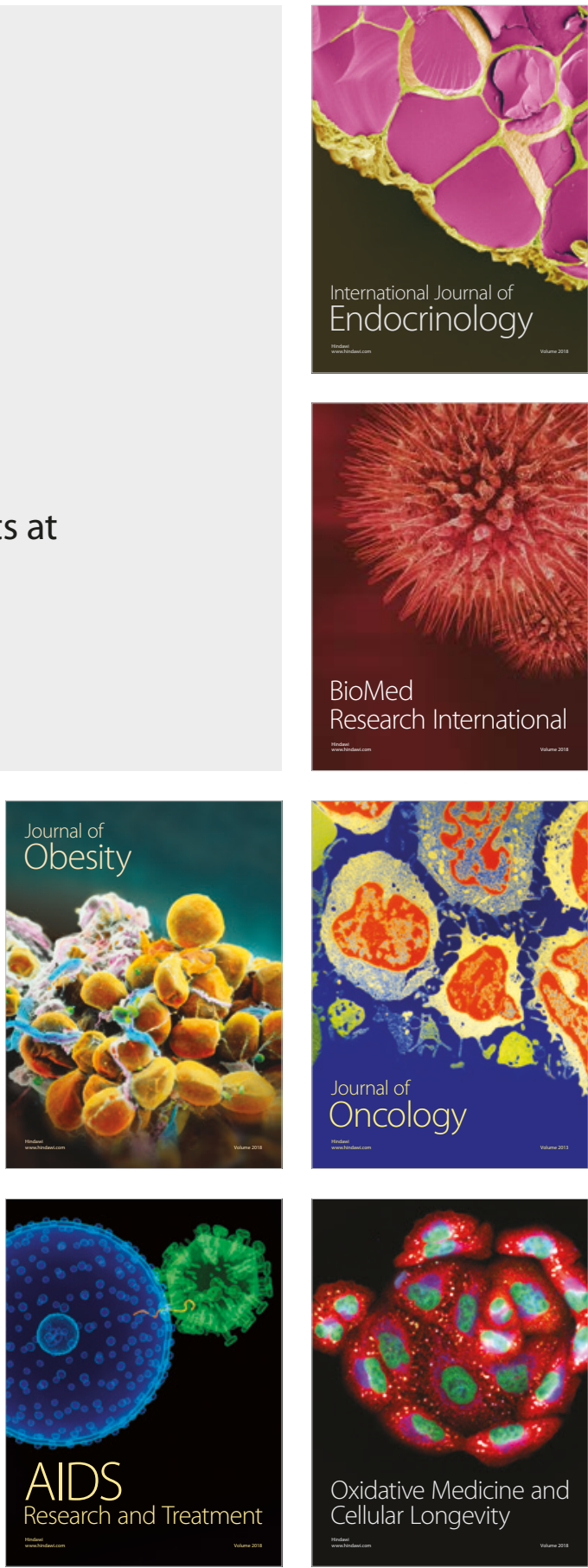